\title{
Tracking Error Modeling in Presence of Satellite Imperfections
}

\author{
Mariano Vergara, Matteo Sgammini, Yuji Zhu, Steffen Thoelert, Felix Antreich \\ German Aerospace Center (DLR), Germany
}

\section{BIOGRAPHY}

Mariano Vergara received the BSc. and the MSc. degrees in Telecommunications engineering from the University of Naples Federico II, Italy, in 2006, and from the Technische Universität Kaiserslautern (TU-KL), Germany, in 2009, respectively. He is currently pursuing a $\mathrm{Ph}$.D. degree at the Universtat Autònoma de Barcelona (UAB), Spain, on signal design for global navigation satellite systems (GNSS). Since 2008, he has been an Associate Researcher with the Department of Navigation, Institute of Communications and Navigation of the German Aerospace Center (DLR).

Matteo Sgammini received the BEng degree in electrical engineering in 2005 from the University of Perugia. He joined the Institute of Communications and Navigation of the German Aerospace Center (DLR) in 2008. His field of research is interference detection and mitigation for global navigation satellite systems (GNSS) .

Yuji Zhu enrolled in the Shanghai Jiaotong University in 2005 and accomplished her bachelor study in 2009. At the moment she pursues a master degree at the Technical University of Munich and she is currently working on ay master thesis about the phase biases in precise point positioning.

Steffen Thoelert received his diploma degree in electrical engineering with fields of expertise in high-frequency engineering and communications at the University of Magdeburg, Germany. Since then he has been with the German Aerospace Centre (DLR). Currently he works within the topics of GNSS verification and system calibration.

Felix Antreich (M'06) received the diploma and the Ph.D. degree in electrical engineering from the Technische Universität München (TUM), Germany in 2003 and 2011, respectively. Since July 2003, he has been an Associate
Researcher with the Department of Navigation, Institute of Communications and Navigation of the German Aerospace Center (DLR). His research interests include sensor array signal processing for global navigation satellite systems (GNSS) and wireless communications, estimation theory and signal design for synchronization, and GNSS.

\begin{abstract}
Modeling both nominal distortions and signal anomalies (i.e. Evil waveforms, ICAO threat models) is crucial for an accurate user error budget for SBAS and GBAS (multifrequency, multi-constellation). Such analysis of particular interest for the ongoing standardization work of L1L5 SBAS services. In this paper we propose a method to jointly estimate the analogue and the digital distortions. This comes very useful in those cases in which the estimates of the two satellite imperfections are interdependent, e.g. when the time-bandwidth product is small. The impact of both satellite imperfections on the ranging performance of a GNSS receiver is assessed by means of analytical formulas which make use of the Cross-Power Spectrum (CPS) between the distorted and the ideal signal, and of the Power Spectral Density (PSD) of the nominal (ideal) signal.
\end{abstract}

\section{INTRODUCTION}

Actual GNSS signals radiated by satellites do not match exactly the ideal GNSS signals defined in the Signal-In-Space official documents. This is due to several non-idealities of the satellite payloads, some of them are deemed acceptable (so called "nominal distortions") and others not (so called "anomalous signal deformations"). It is thus of high importance for several GNSS application to assess the amount of these undesired effects for each satellite of a GNSS constellation, as different satellites may introduce different distortions [1].

The International Civil Aviation Organization (ICAO), through its GNSS Working Group B, has set forth a class 
of signal deformations against which any candidate differential GNSS-based precision approach and landing system must be able to protect its users. These deformations are classified in 3 threat models [2]: threat model A, threat model B and threat model C. Threat model A consists of a non-linear distortion that changes the actual chip duration of the transmitted signal; this distortion is also known as digital distortion. Threat model $\mathrm{B}$ consists of a linear distortion, which accounts for all linear distortions introduced by the satellite payload; this distortion is also known as analog distortion. Thread model $\mathrm{C}$ is the superposition of threat model A and B. The deteriorating effects of these distortion on the ranging performance depend both on the signal type and on the structure of the considered GNSS receiver.

The first contribution of this work lays in proposing a method for jointly estimate the analog and the digital distortion. The second contribution consists in using these estimates as input for a GNSS receiver performance model. No assumption is made on the linear transfer function of the analog distortion, i.e. we do not restrict ourselves to observe linear distortions of a second order system, as recommended in the ICAO document [2]. The reason for that is that the second order system model is not always accurate as other studies have shown [3], and with signals with lower time-banwidth product the second order system model is even more inaccurate.

In this paper we distinguish two models: a satellite payload model and a receiver performance model. The former is meant to model the joint effect of the analogue and the digital distortion on the transmitted signal and the latter is used to assess the effect of the overall signal imperfections on the tracking performance of a GNSS receiver. The error in the tracking performance is characterised both in terms of tracking error bias and tracking error variance. As a case study we considered a B3 Beidou signal and we used a noncoherent early-minus-late power receiver model.

\section{SIGNAL MODEL}

The signal we consider is an interplex [4] made of N DSCDMA signals, which we express with the same formulation used in [5]:

$$
\begin{aligned}
x(t)= & -\sum_{n=2}^{N} g_{n}(\boldsymbol{\theta}) s_{n}(t)+v_{I}(t ; \boldsymbol{\theta}) \\
& \mathrm{j}\left[g_{1}(\boldsymbol{\theta}) s_{1}(t)+v_{Q}(t ; \boldsymbol{\theta})\right]
\end{aligned}
$$

with:

$g_{n}(\boldsymbol{\theta})$ : the weighting factor of the $n$-th signal component. The weighting factors are determined by the interplex angles $\boldsymbol{\theta}=\left[\theta_{1}, \ldots, \theta_{N}\right]$,

$v_{I}(t ; \boldsymbol{\theta})$ : in-phase intermodulatiom (IM) products terms,
$v_{Q}(t ; \boldsymbol{\theta})$ : quad. intermodulatiom (IM) products terms.

The general expression for $v_{I}(t ; \boldsymbol{\theta})$ and $v_{Q}(t ; \boldsymbol{\theta})$ is reported in [6]. The $n$-th DS-CDMA signal consists of

$$
s_{n}(t)=\sum_{m=-\infty}^{\infty} c_{m}^{(n)} p_{n}\left(t-m T_{n}\right)
$$

where $c_{m}^{(n)}$ is the $n$-th chip (eventually spreaded by a data symbol), $T_{n}$ is the $n$-th chip time and $p_{n}(t)$ its chip pulse shape.

For the simple case of a 2-channels interplex, no intermodulation products terms are present, i.e. $v_{I}(t ; \boldsymbol{\theta})=$ $v_{Q}(t ; \boldsymbol{\theta})=0$. So for the special case that we consider in this study,

$$
x(t)=-s_{2}(t)+\mathrm{j} s_{1}(t)
$$

\section{SATELLITE PAYLOAD MODEL}

The satellite payload is modelled as a cascade of two blocks: the first one that accounts for the digital distortion and the second one that is responsible for the analogue distortion. In our model the digital distortion affects the signal components separately and independently, and the analog distortion affect the overall interplex signal. The digital distortion is defined for a real binary signal, i.e. a BPSK signal with an ideal rectangular pulse, i.e. with unlimited bandwidth. The analogue distortion is responsible for the linear filtering of the band-unlimited ideal pulse. The observed interplex signal is the superposition of digital and analog distortions. At receiver side, the tracking is per-

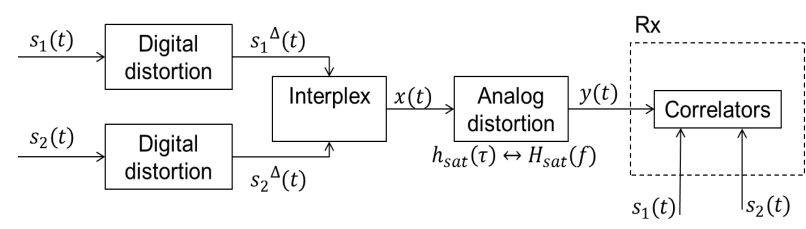

Fig. 1 ..

formed component-wise. For example, in the case of a twochannels interplex with perfect estimate of the carrier phase (coherent detection), the estimated signal components are

$$
\begin{aligned}
& \tilde{s}_{1}(t)=\Im[y(t)] \\
& \tilde{s}_{2}(t)=-\Re[y(t)]
\end{aligned}
$$

These signals are cross-correlated with ideal replicas:

$$
\begin{aligned}
& R_{\tilde{s}_{1} s_{1}}=\frac{1}{T_{0}} \int_{T_{0}} \tilde{s}_{1}(t) s_{1}(t+\tau) \mathrm{d} t \\
& R_{\tilde{s}_{2} s_{2}}=\frac{1}{T_{0}} \int_{T_{0}} \tilde{s}_{2}(t) s_{2}(t+\tau) \mathrm{d} t,
\end{aligned}
$$


with $T_{0}$ indicating the period (or code epoch duration) of the signal components. In the case of coherent detection these cross-correlations are real, but with a non-coherent detection these cross-correlations are complex. The impulse response of the analog distortion is in general complex, i.e. the transfer function is non-hermitian. The impact of the non-hermitianity of the linear (analog distortion) is felt even if the codes are perfectly uncorrelated and the carrier phase is estimated perfectly. Indeed, even assuming perfect carrier phase recovery (coherent), we have that

$$
\begin{aligned}
& \hat{s}_{1}(t)=x^{R}(t) \otimes h_{\text {sat }}^{R}(t)-x^{I}(t) \otimes h_{\text {sat }}^{I}(t) \\
& \hat{s}_{2}(t)=x^{I}(t) \otimes h_{\text {sat }}^{R}(t)+x_{R}(t) \otimes h_{\text {sat }}^{I}(t)
\end{aligned}
$$

with $x^{R}(t)$ and $h_{\text {sat }}^{R}(t)$ indicating the real parts of the interplex signal and the impulse response respectively and with $x^{I}(t)$ and $h_{\text {sat }}^{I}(t)$ indicating their imaginary parts.

\subsection{Digital distortion}

By digital distortion it is meant a variation of the chip duration with respect to the the nominal chip duration, i.e. the inverse of the chip rate. The digital distortion is defined for band-unlimited signals. The problem in correctly estimating the digital distortion is that we do not observe the band-unlimited signal but a filtered version of the digitally distorted signal. A band-limitation of a digitally distorted signal - and more in general a linear filtering - changes the location of the zero crossings of the signal on the interplex channels. This makes the estimate of the variation of the actual chip duration dependent on the linear distortion that the transmitted signal has gone through.

The digital distortion is also known as ICAO threat model A [2]. The digital distortion causes flat spots or plateaus atop the cross-correlation peak [1], and so a unique maximum point of the cross-correlation function cannot be determined. Consequently, the discriminator curve is distorted and it does not cross the zero at only one point, but it is identically zero within a certain interval of the tracking error. In theory, such a signal is not trackable as no stable tracking point exist. Nevertheless, when the digital distortion is connected with an analog distortion - i.e. linear filtering - and in particular when the bandwidth of the analog filter is small enough, then the cross-correlation function can have a unique maximum and the tracking is possible. In the following we will indicate the generic ideal signal component by $s(t)$ and its digitally distorted counterpart by $s_{\Delta}(t)$. We shall indicate with $\eta$ the digital distortion in terms of $T_{c}$. In general $\eta$ is a random variable and this makes the digital distortion a stochastic non-linear distortion. In order to simplify the calculations, in this study we shall model $\eta$ as a parameter, instead as a random variable. In other terms we shall assume that the digital distortion is deterministic and constant throughout the observed signal.
Nevertheless, it is to be kept in mind that in general the digital distortion is a stochastic (non-linear) transformation of the ideal signal.

The PSD of a digitally distorted signal was calculated by [7]:

$$
\Phi_{s_{\Delta} s_{\Delta}}(f)=\psi_{\text {continuous }}(f)+\psi_{\text {discrete }}(f)
$$

where $\psi_{\text {continuous }}(f)$ and $\psi_{\text {discrete }}(f)$ are the continuous and the discrete components of the PSD of a digitally distorted signal indicated in Eq. (2.53) in [8, p. 63]. Nevertheless what determines the performance of the tracking is not the autocorrelation of the transmitted signal, but rather its cross-correlation with the ideal replica generated in the receiver. We will first model this cross-correlation in absence of analog distortion, and in the next section we will add the effect of the analog distortion. The cross-correlation between a digital distorted signal and the ideal signal (no analog distortion) in presence or a rectangular chip pulse shape can be modelled as a piecewise linear function:

$$
R_{s_{\Delta} s}(\tau)= \begin{cases}\frac{\frac{\tau}{T c}+1+\eta}{2}, & -(1+\eta) T_{c}<\tau<T_{c} \\ \frac{\tau}{T c}+1+\frac{\eta}{2}, & -T_{c}<\tau<-\eta T_{c} \\ 1-\frac{\eta}{2} & -\eta T_{c}<\tau<0 \\ 1-\frac{\eta}{2}-\frac{\tau}{T c}, & 0<\tau<T_{c}(1-\eta) \\ \frac{1-\frac{\tau}{T c}}{2}, & T_{c}(1-\eta)<\tau<T_{c}\end{cases}
$$

This cross-correlation is normalised such that its maximum is 1 when there is no digital distortion $(\eta=0)$. For $|\eta|>0$ there is a correlation loss. The cross-power spectral density between the digitally distorted signal and the ideal signal is the Fourier Transform of this cross-correlation:

$$
\Phi_{s_{\Delta} s}(f)=\mathcal{F}\left[R_{s_{\Delta} s}(\tau)\right]
$$

Note that as the ideal signal component $s(t)$ and its digitally distorted counterpart $s_{\Delta}(t)$ are both real, the CPS (10) is thus hermitian. In this paper we neglect any effect that the digital distortion on one channel has on other interplex channels.

\subsection{Analog distortion}

The analogue distortion accounts for all the linear distortion of the satellite payload.

In general the impulse response of the satellite can be complex, as in Eq. (7). In frequency domain this corresponds to a non-hermitian transfer function. The effect of the imaginary part of the impulse response is to introduce crosstalk among the signal component, even with coherent detection with a perfect estimate of the carrier phase or with a non-coherent detection. This cross-talk increases the 
MAI(Multiple Access Interference). This increase of the MAI depends on the power contained in the imaginary part of the impulse response (i.e. anti-hermitian component of the transfer function), the interplex order, and the decorrelation performance of the spreading codes. The presence of an imaginary component in the impulse response (i.e. of an anti-hermitian component in the transfer function) has a degrading effect on the GNSS receiver performance performing a component-wise tracking, even if the spreading codes of the different signal components are assumed perfectly orthogonal. As Eq. (7) shows, part of the power of the desired signal is lost because it "spills" on another signal component. The amount of lost power depends on the energy contained in the imaginary part of the impulse response:

$$
\frac{\int_{-\infty}^{\infty}\left|h_{\text {sat }}^{I}(\tau)\right|^{2} \mathrm{~d} \tau}{\int_{-\infty}^{\infty}\left|h_{\text {sat }}(\tau)\right|^{2} \mathrm{~d} \tau}
$$

In the ICAO specifications [2] a second order model is proposed. Nevertheless, as already noted in [3], this model is not always adequate. On top of that, when the timebandwidth product is small (e.g. in the L5 band), the ICAO second order system model is even more inaccurate. To a second order system model corresponds an hermitian transfer function, so power losses caused by possible non-hermitianities of the transfer function are taken into account. In the following we will not make any a-priori assumption on the linear transfer function responsible for the analog distortion.

The linear distortion caused by the satellite must be distinguished from the additional linear distortion caused by the receiver front-end filter. To this end, let the receiver front-end filter be indicated by $H_{R x}(f)$. The normalised cross-correlation between the incoming distorted interplex signal and the local replica is

$R_{\hat{s} s}(\tau)=\frac{\int_{-\infty}^{\infty} \Phi_{s_{\Delta} s}(f) H_{s a t}(f) H_{R x}^{*}(f) e^{\mathrm{j} 2 \pi f \tau} \mathrm{d} f}{\sqrt{\int_{-\infty}^{\infty} \Phi_{s_{\Delta} s_{\Delta}}(f)\left|H_{s a t}(f)\right|^{2}\left|H_{R x}(f)\right|^{2} \mathrm{~d} f} \sqrt{\int_{-\infty}^{\infty} \Phi_{s s}(f) \mathrm{d} f}}$

where $\Phi_{s s}(f)$ is the PSD of the signal component (2), $\Phi_{s_{\Delta} s}(f)$ is the CPS between the digitally distorted signal and nominal signal (local replica). This autocorrelation is in general complex. By a coherent detection, this crosscorrelation is real and the corresponding CPS hermitian.

\subsection{Receiver model}

The tracking is performed component-wise by projecting the interplex signal on the desired signal component. We shall indicate the desired signal component by $s(t)$. The spreading codes will be assumed perfectly orthogonal, so the cross-talk among the signal components is neglected. The tracking performance of the receiver is modelled as in [9], where the following assumptions are made:

- Steady-state tracking: the DLL is already tracking and the joint effect of distortions plus noise do not cause a loss of lock.

- Small tracking jitter: The tracking point oscillates around the lock point in a restricted set of values, for which the composite discriminator is approximately linear.

- Proper gaussian noise [10, p. 26]: the noise samples at the output of the correlator on the I and Q branched are uncorrelated.

The composite discriminator is the the S-curve that results from channel impairments or, as in the case of this paper, by distortions introduced by the satellite. The composite discriminator is calculated from the cross-correlation (12). The tracking performance is assessed in terms of tracking error bias and tracking error variance. The tracking error is indicated by $\varepsilon$. The tracking error bias is calculated as the value of the tracking error at which the composite discriminator is zero:

$$
b=\underset{\varepsilon}{\arg }\left\{S_{c}(\varepsilon)=0\right\}
$$

The variance is calculated as follows

$$
\begin{aligned}
\sigma_{\varepsilon}^{2} & =\frac{N_{T}(0) 2 B_{L}}{P^{2}\left[S_{c}^{\prime}(b)\right]^{2}} \\
& =\frac{2 B_{L} N_{0}}{P} \frac{4\left|R_{\hat{s} s}(\Delta)\right|^{2}\left(1-R_{p}(2 \Delta)\right)}{\left[S_{c}^{\prime}(b)\right]^{2}}+\frac{2 B_{L} N_{0}}{P} \frac{N_{0}}{P T_{p}} \frac{\left(2-2 R_{p}(2 \Delta)\right)}{\left[S_{c}^{\prime}(b)\right]^{2}} \\
& =\frac{1}{\rho_{\text {loop }}} \frac{4\left|R_{\hat{s} s}(\Delta)\right|^{2}\left(1-R_{p}(2 \Delta)\right)}{\left[S_{c}^{\prime}(b)\right]^{2}}+\frac{1}{\rho_{\text {loop }}} \frac{1}{\rho_{\text {post }}} \frac{\left(2-2 R_{p}(2 \Delta)\right)}{\left[S_{c}^{\prime}(b)\right]^{2}}
\end{aligned}
$$

where:

$\Delta$ : one-sided correlator spacing of the DLL,

$R_{p}(\varepsilon)$ : auto-correlation function of the chip pulse shape of the ideal signal component (2),

$N_{T}(0)$ : DC value of the PSD of the noise after the correlation,

$P$ : received power,

$T_{p}$ : integration time,

$B_{L}$ : loop bandwidth.

Note that while for the local replica a deterministic chip pulse shape can be defined, this is not the case for the incoming signal affected by digital distortion. The reason for that is that the digital distortion is a stochastic non-linear distortion. As a consequence of that, a deterministic crosscorrelation between the chip of the incoming signal and the chip of the local replica cannot be defined. In other terms, the cross-correlation between the incoming signal and the local replica cannot be factorised into the cross-correlation between the spreading codes and the cross-correlation between the pulse shapes $[11$, p. 125]. The expression of the 
discriminator gain of a non-coherent DLL is reported in the appendix. Note that since in general the tracking error bias is not null, the discriminator gain is not to be calculated at zero but rather at the bias point, as explained in [9].

\section{ESTIMATION METHOD}

In this section we explain how we estimate the analog and digital distortion from calibrated measurement data. The novelty with respect to previous work is that the estimate is performed jointly, in order to avoid possible coupling effects between the two distortions.

The signal radiated by the satellite is recorded with a measurement system that is extensively and very accurately calibrated. The Doppler shift is estimated and removed. The observable data can be expressed as

$$
\tilde{y}(t)=y(t)+\epsilon_{a t m}(t)+\epsilon_{\text {meas }}(t)+n(t)
$$

with

$\epsilon_{\text {atm }}(t)$ : Atmospheric signal deformations,

$\epsilon_{\text {meas }}(t)$ : Measurement system behavior/deformations,

$n(t)$ : thermal noise.

The terms $\epsilon_{a t m}(t), \epsilon_{\text {meas }}(t)$, and $n(t)$ cause the observed raw data to be not exactly as the signal radiated by the satellite. Nevertheless, Using a high gain antenna with approx. $50 \mathrm{~dB}$ gain in the L-band the noise can be neglected; furthermore it can be assumed that atmospheric (especially ionospheric) distortion in the middle European region is small and consequently negligible or it can be corrected by appropriate models. Thus, it can be stated that the transmitted signal of the satellite is very close to the measured and calibrated signal.

Let the signal $y(t)$ and $\tilde{y}(t)$ be represented by

$$
\begin{aligned}
& \mathbf{y}=\left[y_{1}, y_{2}, \ldots, y_{L_{y}}\right]^{\mathrm{T}} \in \mathbb{C}^{L_{y} \times 1}, \\
& \tilde{\mathbf{y}}=\left[\tilde{y}_{1}, \tilde{y}_{2}, \ldots, \tilde{y}_{L_{y}}\right]^{\mathrm{T}} \in \mathbb{C}^{L_{y} \times 1},
\end{aligned}
$$

and let the impulse response be vectorised as follows

$$
\mathbf{h}=\left[h_{1}, h_{2}, \ldots, h_{L_{h}}\right]^{\mathrm{T}} \in \mathbb{C}^{L_{h} \times 1} .
$$

The digital distortion present on each signal component is measured in terms of chip time. The digital distortion can be both positive and negative, and it cannot be in magnitude larger than one chip. Let us arrange the digital distortions present on the $\mathrm{N}$ signal components in a vector (in our case $\mathrm{N}=2$ ):

$$
\boldsymbol{\eta}=\left[\eta_{1}, \eta_{2}\right]^{\mathrm{T}} \quad \eta_{1}, \eta_{2} \in[-1,1]
$$

The ideal undistorted interplex signal is

$$
\mathbf{x}(\boldsymbol{\eta})=\left[x_{1}, x_{2}, \ldots, x_{L_{y}}\right]^{\mathrm{T}} \in \mathbb{C}^{L_{y} \times 1}
$$

and let $\mathbf{X}(\boldsymbol{\eta})$ indicate the $L_{y} \times L_{h}$ convolution matrix with respect to vector $\mathbf{x}(\boldsymbol{\eta})$.

Using these measurement data and these definitions the estimation method can be explained very briefly. The estimation problem can be formulated as

$$
(\hat{\boldsymbol{\eta}}, \hat{\mathbf{h}})=\underset{\boldsymbol{\eta}, \mathbf{h}}{\operatorname{argmin}}\left\{\|\tilde{\mathbf{y}}-\mathbf{X}(\boldsymbol{\eta}) \mathbf{h}\|_{2}^{2}\right\}
$$

with

$\|\tilde{\mathbf{y}}-\mathbf{X}(\boldsymbol{\eta}) \mathbf{h}\|_{2}^{2}=(\tilde{\mathbf{y}}-\mathbf{X}(\boldsymbol{\eta}) \mathbf{h})^{\mathrm{H}}(\tilde{\mathbf{y}}-\mathbf{X}(\boldsymbol{\eta}) \mathbf{h})=\boldsymbol{\Lambda}(\boldsymbol{\eta}, \mathbf{h})$

Based on the measured and calibrated data the chip sequences of the in-phase and quadrature signal component have been detected. Doing so, the above mentioned minimization problem is solved by differentiating $\Lambda(\boldsymbol{\eta}, \mathbf{h})$ with respect to $\mathbf{h}^{\mathrm{H}}$ and substituting it in (22) and equating to zero. This results to

$$
\hat{\boldsymbol{\eta}}=\underset{\boldsymbol{\eta}}{\operatorname{argmin}}\left\{\tilde{\mathbf{y}}^{\mathrm{H}} \mathbf{X}(\boldsymbol{\eta})\left(\mathbf{X}^{\mathrm{H}}(\boldsymbol{\eta}) \mathbf{X}(\boldsymbol{\eta})\right)^{-1} \mathbf{X}^{\mathrm{H}}(\boldsymbol{\eta}) \tilde{\mathbf{y}}\right\}
$$

The estimate of the transfer function can be calculated based on the determined digital distortions:

$$
\hat{\mathbf{h}}=\left(\mathbf{X}^{\mathrm{H}}(\hat{\boldsymbol{\eta}}) \mathbf{X}(\hat{\boldsymbol{\eta}})\right)^{-1} \mathbf{X}^{\mathrm{H}}(\hat{\boldsymbol{\eta}}) \tilde{\mathbf{y}}
$$

\section{STUDY CASE: BEIDOU B3 SIGNAL}

For a study case we picked the Beidoud B3 signal. The Beidoud M3 signal consist of an interplex signal with two signal components, each with a chip rate of $10.23 \mathrm{MHz}$, modulated by a carrier frequency of $1268,52 \mathrm{MHz}$. After having compensated the Doppler and other abovementioned effects, the PSD of the received signal is depicted in Fig.2, nwhile the IQ constellation is depicted in Fig.3. In Fig. 2 are to be observed spectral lines due to the digital distortion, according to the model provided in [7].

The estimated transfer function is plotted in Fig. 4 and its equivalent impulse response in Fig.5. As it can be observed, the impulse response contains some power in the imaginary part, and reflecting the fact that the frequency response is not hermitian.

The estimate of the digital distortion on the two signal components are $2 \%$ and $1.5 \%$ :

$$
\boldsymbol{\eta}=[0.02,0.015]
$$

The receiver tracking performance in presence of the estimated signal distortions is calculated with the parameters in Table 1, and for different values of correlator spacing and receiver bandwidth. In Fig.7 and Fig.8 the tracking error jitter and the tracking error bias are represented respectively. As a comparison term, also the tracking jitter in ideal 


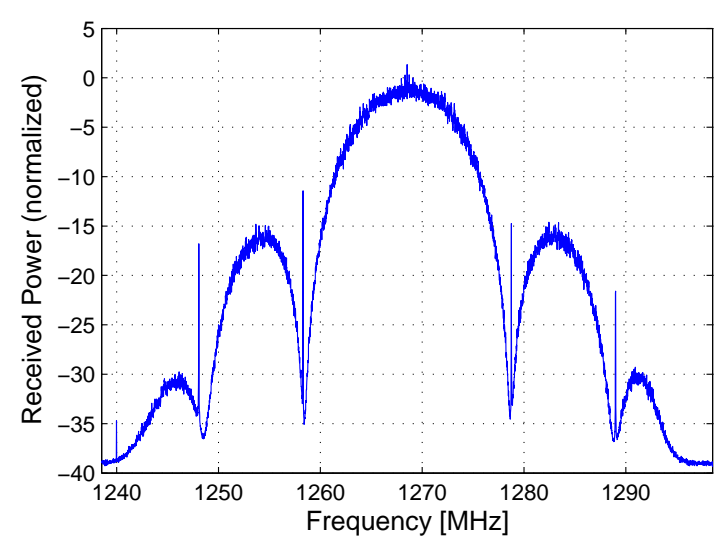

Fig. 2 Beidou M3 (NORAD: 38250) - B3-Band - Spectra - 14 June 2012

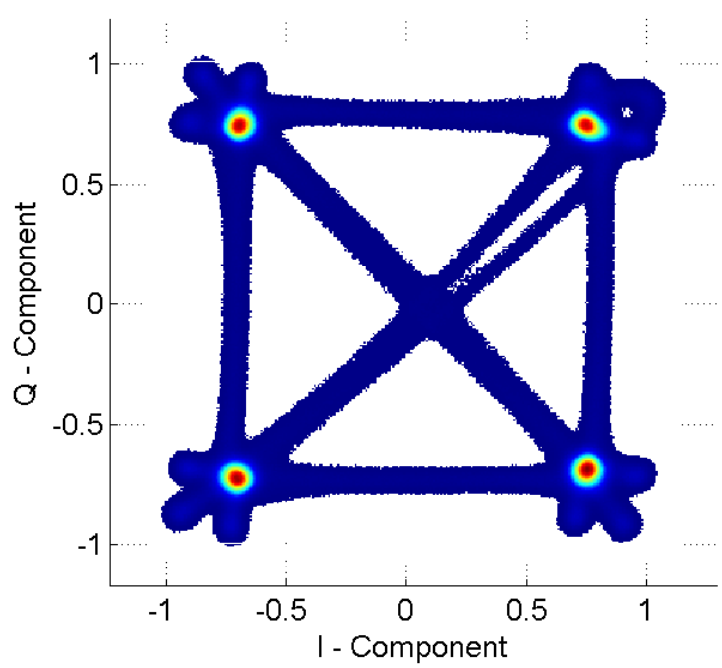

Fig. 3 Beidou M3 (NORAD: 38250) - B3-Band - IQ constellation - 14 June 2012

conditions is shown (Fig. [?]). Worth of notice is that when analog and digital distortions impact the signal, the optimal pair of correlator spacing and receiver bandwidth changes. In particular, in presence of satellite imperfections it cannot be said any more that by increasing the bandwidth and decreasing the correlator spacing the variance of the tracking error decreases. Most importantly, these results show that characterising the tracking error only in terms of bias is not adequate, as it can even happen that for a certain combination of receiver bandwidth and correlator spacing the tracking error bias is zero.

\section{CONCLUSIONS}

In this paper we have approached the problem of estimating the digital and analog distortions of a GNSS satellite and have modeled their impact on the performance of a
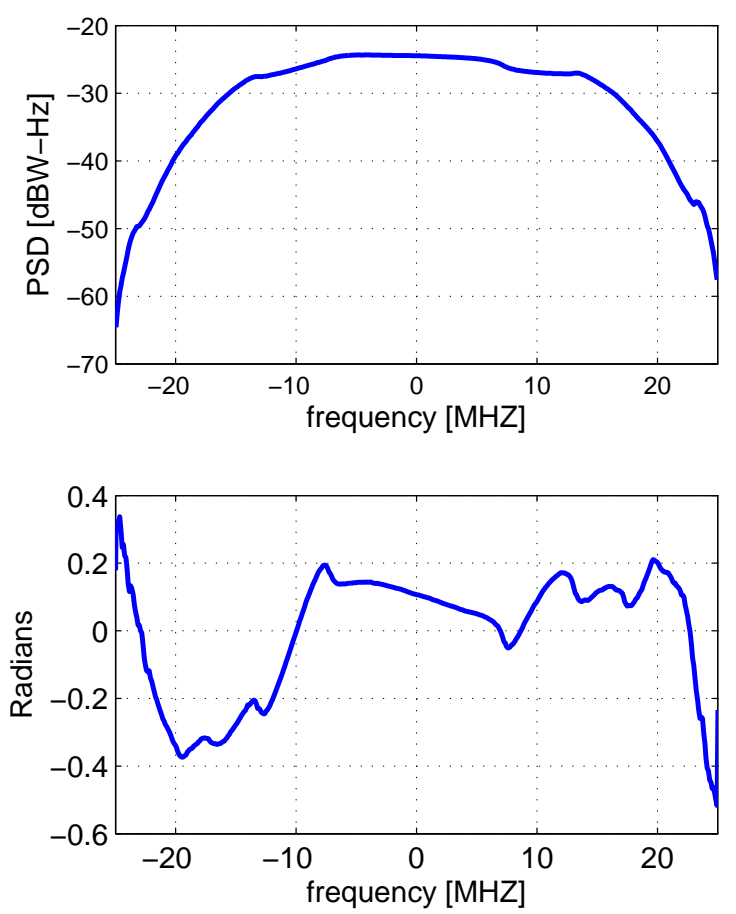

Fig. 4 Amplitude spectrum and phase spectrum of the estimated frequency reponse

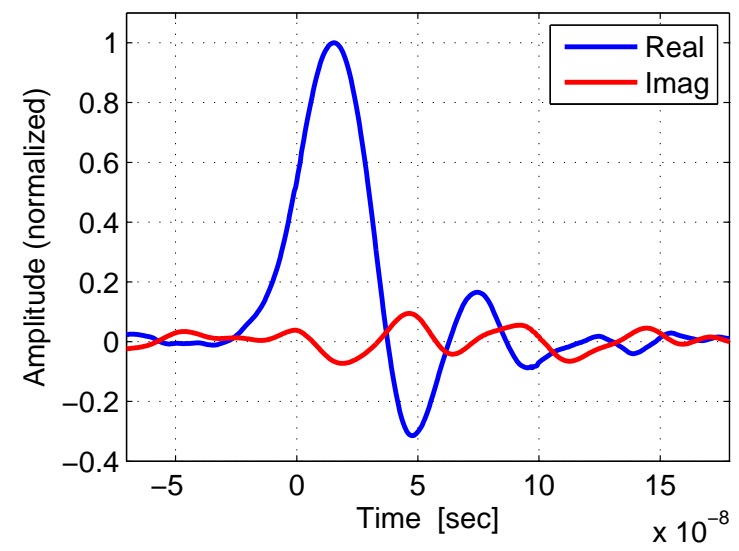

Fig. 5 Estimated impulse response

GNSS receiver. The first element of novelty is that we estimated analog and digital distortion in a joint fashion. This is important as in some cases - in particualr when the signal time-bandwidth product is small - the estimates of the two kinds of signal deformations become interdependent. These estimates of the digital and analog distortions has been calculated starting from real measurements obtained with a high gain directional antenna.

A second element of novelty is that we did not limit to assess the tracking error bias, but rather we provided a more complete performance model that allows to calculate the 


\begin{tabular}{|c|c|}
\hline Parameter & Parameter value \\
\hline Tracked signal & Beidou B3-I \\
DLL type & Non-Coherent \\
$\frac{C}{N_{0}}$ & $40 \mathrm{~dB}$ \\
Integration time & $1 \mathrm{~ms}$ \\
Loop Bandwidth & $1 \mathrm{~Hz}$ \\
\hline
\end{tabular}

Table 1 Tracking parameters.

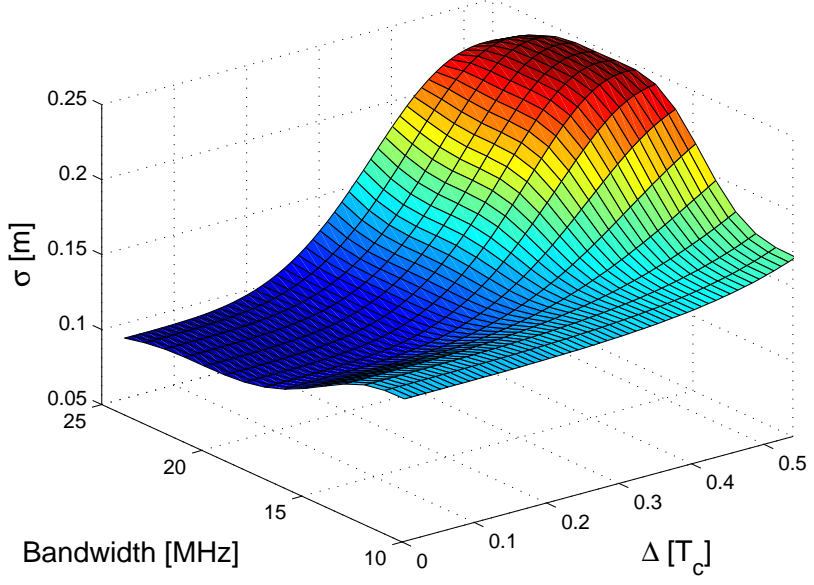

Fig. 6 Tracking error jitter in nominal conditions for different bandwidth and correlator spacings. Only one signal component is tracked. Rx parameters in Table I.

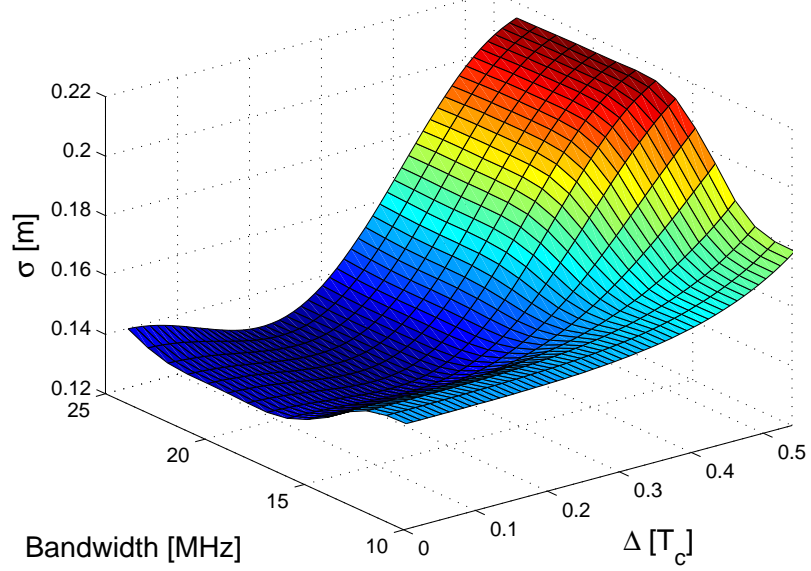

Fig. 7 Tracking error jitter in presence of analog and diigital distortions for different bandwidth and correlator spacings. Only one signal component is tracked. Rx parameters in Table I.

joint effect of analog plus digital distortion in terms of tracking error bias and tracking error variance (or jitter). This performance model has been with software simulations (Montecarlo runs) of a GNSS receiver.

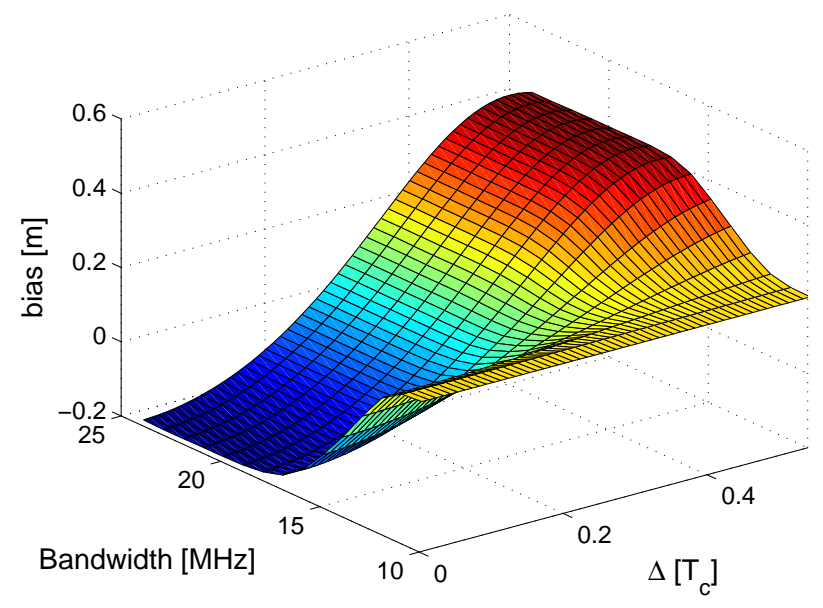

Fig. 8 Tracking error bias in presence of analog and diigital distortions for different bandwidth and correlator spacings. Only one signal component is tracked. Rx parameters in Table I.

The technical relevance of the paper consist in providing a mathematical tool to assess the impact of both nominal distortions and anomalous signal deformations on the performance of a GNSS receiver.

\section{APPENDIX}

In this appendix we report the discriminator gain for a noncoherent DLL in signal distortion. The calculation holds also for other impairments as multipath, interference, and matched filter that are not perfectly match to the incoming chip pulse shape.

We define the equivalent CPS as:

$$
\Psi(f)=\Phi_{s_{\Delta} s}(f) H_{s a t}(f) H_{R x}(f)=\mathcal{F}\left[R_{\hat{s} s}(\tau)\right]
$$

where in the general case $R_{\hat{s} s}(\tau)$ indicates the crosscorrelation between the incoming signal that it is desired to track and its ideal replica. $\Psi(f)$ is the Fourier representation of such cross-correlation. Moreover let us decompose this CPS in

$$
\begin{aligned}
\Psi^{\mathcal{R}, \text { even }}(f) & =\frac{\Psi^{\mathcal{R}}(f)+\Psi^{\mathcal{R}}(-f)}{2} \\
\Psi^{\mathcal{R}, \text { odd }}(f) & =\frac{\Psi^{\mathcal{R}}(f)-\Psi^{\mathcal{R}}(-f)}{2} \\
\Psi^{\mathcal{I}, \text { odd }}(f) & =\frac{\Psi^{\mathcal{I}}(f)+\Psi^{\mathcal{I}}(-f)}{2} \\
\Psi^{\mathcal{I}, \text { even }}(f) & =\frac{\Psi^{\mathcal{I}}(f)+\Psi^{\mathcal{I}}(-f)}{2}
\end{aligned}
$$




$$
\begin{aligned}
& S_{c}^{\prime}(b)=2\left[\int_{-B}^{B} \Psi^{\mathcal{R}, \text { even }}(f) \cos (2 \pi f(b-\Delta)) \mathrm{d} f\right. \\
& \left.-\int_{-B}^{B} \Psi^{\mathcal{I}, o d d}(f) \sin (2 \pi f(b-\Delta)) \mathrm{d} f\right] \times \\
& {\left[-\int_{-B}^{B} \Psi^{\mathcal{R}, \text { even }}(f)(2 \pi f) \sin (2 \pi f(b-\Delta)) \mathrm{d} f\right.} \\
& \left.-\int_{-B}^{B} S_{x y}^{\mathcal{I}, o d d}(f)(f)(2 \pi f) \cos (2 \pi f(b-\Delta)) \mathrm{d} f\right] \\
& +2\left[\int_{-B}^{B} \Psi^{\mathcal{R}, o d d}(f) \sin (2 \pi f(b-\Delta)) \mathrm{d} f\right. \\
& \left.+\int_{-B}^{B} \Psi^{\mathcal{I}, \text { even }}(f) \cos (2 \pi f(\varepsilon-\Delta)) \mathrm{d} f\right] \times \\
& {\left[\int_{-B}^{B} \Psi^{\mathcal{R}, o d d}(f)(2 \pi f) \cos (2 \pi f(b-\Delta)) \mathrm{d} f\right.} \\
& \left.-\int_{-B}^{B} \Psi^{\mathcal{I}, \text { even }}(f)(2 \pi f) \sin (2 \pi f(b-\Delta)) \mathrm{d} f\right] \\
& -2\left[\int_{-B}^{B} \Psi^{\mathcal{R}, \text { even }}(f) \cos (2 \pi f(b+\Delta)) \mathrm{d} f\right. \\
& \left.-\int_{-B}^{B} \Psi^{\mathcal{I}, \text { odd }}(f) \sin (2 \pi f(\varepsilon+\Delta)) \mathrm{d} f\right] \times \\
& {\left[-\int_{-B}^{B} \Psi^{\mathcal{R}, \text { even }}(f)(2 \pi f) \sin (2 \pi f(b+\Delta)) \mathrm{d} f\right.} \\
& \left.-\int_{-B}^{B} \Psi^{\mathcal{I}, \text { odd }}(f)(2 \pi f) \cos (2 \pi f(b+\Delta)) \mathrm{d} f\right] \\
& -2\left[\int_{-B}^{B} \Psi^{\mathcal{R}, o d d}(f) \sin (2 \pi f(b+\Delta)) \mathrm{d} f\right. \\
& \left.+\int_{-B}^{B} \Psi^{\mathcal{I}, \text { even }}(f) \cos (2 \pi f(b+\Delta)) \mathrm{d} f\right] \times \\
& {\left[\int_{-B}^{B} \Psi^{\mathcal{R}, o d d}(f)(2 \pi f) \cos (2 \pi f(b+\Delta)) \mathrm{d} f\right.} \\
& \left.-\int_{-B}^{B} \Psi^{\mathcal{I}, \text { even }}(f)(2 \pi f) \sin (2 \pi f(b+\Delta)) \mathrm{d} f\right]
\end{aligned}
$$

\section{REFERENCES}

[1] R. Eric Phelts and Dennis M. Akos, "Effects of signal deformations on modernized gnss signals," Journal of Global Positioning Systems, vol. 5, no. 1, pp. 2-10, 2006.

[2] International Civil Aviation Organization (ICAO), International standards and recom-mended practices, November 2001.

[3] Robert Eric Phelts, Todd Walter, and Per Enge, "Characterizing Nominal Analog Signal Deformation on GNSS Signals," in Proceedings of the 22nd International Technical Meeting of The Satellite Division of the Institute of Navigation (ITM 2009), Savannah, GA, September 2009.

[4] S.Butman and Uzi Timor, "Interplex: An efficient Multichannel PSK/PM Telemetry System," IEEE Trans. on Communications, vol. 20, no. 3, pp. 415419, June 1972.
[5] M. Vergara and F. Antreich, "Power efficient evolution of the interplex scheme," in International Technical Meeting ITM 2013, San Diego, USA, January 2013, Institute Of Navigation (ION).

[6] M. Vergara, F. Antreich, G.Liva, and B. Matuz, "Multi-Service Data Dissemination for Space-based Augmentation Systems," in proceedings of IEEE Aerospace 2013, Big Sky Montana, USA, March 2013.

[7] Dennis F. Bishop, Samson Million, Tien M. Nguyen, and Marvin K. Simon, "The Power Spectrum of Unbalanced NRZ and Biphase Signals in the Presence of Data Asymmetry," IEEE Transactions on Electromagnetic Compatibility, vol. 40, no. 1, pp. 55-62, February 1998.

[8] M.K. Simon, S.M. Hinedi, and W.C. Lindsey, Digital Commuications Techniques, Pearson Education, Inc, 1995.

[9] M. Vergara, F. Antreich, and M. Meurer, "Effect of Multipath on Code-Tracking Error Jitter of a Delay Locked Loop," in Fourth European Workshop on GNSS signals and signal processing, GNSS Signal 2009, Wessling, Germany, December 2009.

[10] Peter J. Schreier and Louis L. Scharrf, Statistical Signal Processing of Complex-valued Data, Cambridge university press, 2010.

[11] Felix Antreich, Array Processing and Signal Design for Timing Synchronization, Ph.D. thesis, Technische Unviersitaet Muenchen, 2011. 